\title{
Muscle Weakness Right-Sided, CTCAE
}

National Cancer Institute

\section{Source}

National Cancer Institute. Muscle Weakness Right-Sided, CTCAE. NCI Thesaurus. Code C143683.

A disorder characterized by a reduction in the strength of the muscles on the right side of the body. 